\title{
Optimally locating a junction point for an underground mine to maximise the net present value
}

\author{
K. G. Sirinanda $\quad$ M. Brazil ${ }^{2} \quad$ P. A. Grossman ${ }^{3}$ \\ $\begin{array}{ll}\text { J. H. Rubinstein } & \text { D. A. } \text { Thomas }^{5}\end{array}$
}

(Received 16 December 2013; revised 10 July 2014)

\begin{abstract}
A review of the relevant literature identified an opportunity to develop algorithms for designing the access and construction schedule for an underground mine to maximise the net present value (NPV). The methods currently available perform the optimisation separately. However, this article focuses on optimising the access design and construction schedule simultaneously to yield a higher NPV. Underground mine access design was previously studied with the objective of minimising the haulage and development costs. However, when scheduling is included, time value of money has a crucial effect on locating the
\end{abstract}

http://journal.austms.org.au/ojs/index.php/ANZIAMJ/article/view/7791 gives this article, (C) Austral. Mathematical Soc. 2014. Published August 1, 2014, as part of the Proceedings of the 11th Biennial Engineering Mathematics and Applications Conference. ISSN 1446-8735. (Print two pages per sheet of paper.) Copies of this article must not be made otherwise available on the internet; instead link directly to this URL for this article. 
junction points (Steiner points) in the access network for maximum value. This article proposes an efficient algorithm to optimally locate a single junction, given a surface portal and two ore bodies, for maximum NPV where NPV includes the value of the ore bodies and the construction costs. We describe the variation in the location of the junction for a range of discount rates.

\section{Contents}

1 Introduction

C316

2 Problem formulation

C318

3 The discounted Steiner point algorithm

C322

4 Numerical trials

C323

5 Conclusion and future research

C326

References

C327

\section{Introduction}

The mining industry does not have reliable, accurate or well established algorithms for simultaneously designing the access network for an underground mine and scheduling its construction. Current industry practice is to design the underground access first and then to complete the scheduling. One problem with this process is that the costs of access construction are not reflected in the net present value (NPV) calculation. The NPV is the usual measure of value in the mining industry. Until now underground mine access was not designed with the aim of maximising the NPV. We will first define 
the term NPV and then model the underground mine access design problem as a tree network problem.

Money has a time value and it needs to be analysed in terms of a series of cash flows. Cash flows are brought forward in time with an appropriate discount factor. The discount rate is the key parameter of the process and used to discount future cash flows to the present time. The summation of all the discounted cash flows is called the net present value.

In 1988, Lane [1] expressed the problem of maximising the NPV of a mine as an optimisation problem and the NPV as the sum of the variable and fixed costs. Lane treated mineralised material as a resource. He observed that the resources are actually finite and sooner or later they will be depleted.

We represent the underground access network design problem as a tree network problem, where the locations of the ore resource points are given and the junction points of the network are to be obtained for a specific given objective function. To find a minimum length network connecting a set of given terminals or points, Steiner points or junctions are added to reduce the length. This is the classical Steiner problem [2].

Brazil et al. [3, 4] studied underground mine access design processes and described how to locate the Steiner point. The objective of the problem they analysed was to minimise the infrastructure and haulage costs of an underground mine. However, they did not take the discounted cost into account in their model and have not studied the problem of locating the Steiner point with the objective of maximising the NPV.

The problem we analyse is different from the classical Steiner problem and also from the one discussed by Brazil and Thomas [3], as here the Steiner point is located to maximise the NPV. A Steiner point in a maximum NPV network is a discounted Steiner point. The objective is to locate a single discounted Steiner point to maximise the NPV. 


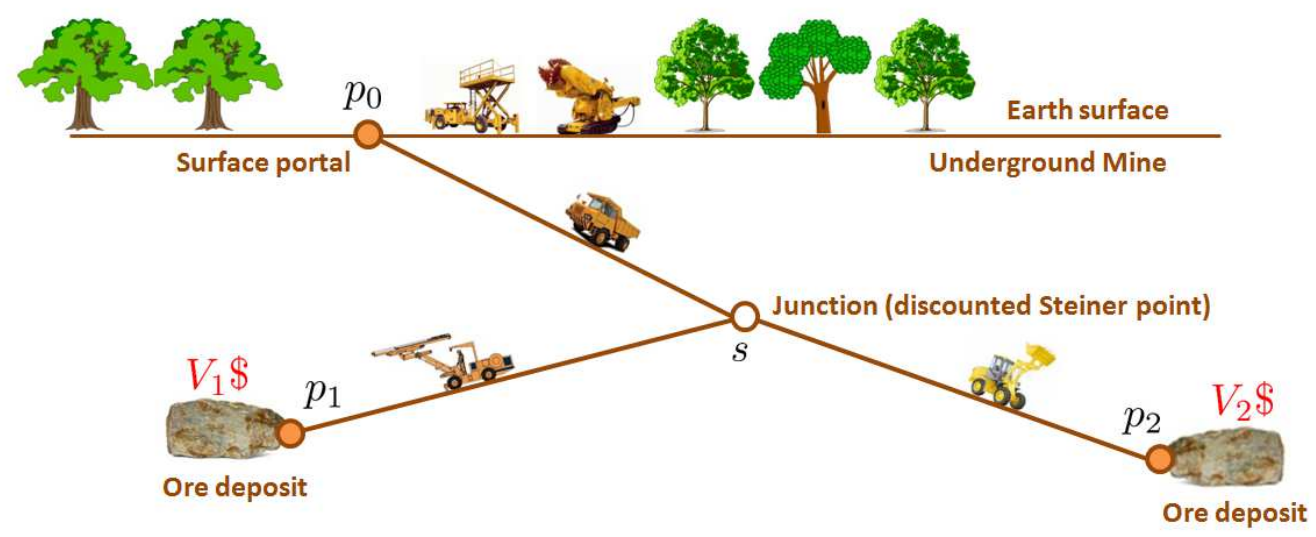

Figure 1: A schematic representation of a simple underground mine.

\section{Problem formulation}

In this section, we formulate and describe the optimisation problem. Figure 1 shows the access network for a simple underground mine. Let $p_{0}=\left(x_{0}, y_{0}, z_{0}\right)$, $p_{1}=\left(x_{1}, y_{1}, z_{1}\right)$ and $p_{2}=\left(x_{2}, y_{2}, z_{2}\right)$ be the surface portal (or breakout point from existing infrastructure) and the access points for ore deposits with worth $\$ V_{1}$ and $\$ V_{2}$, respectively. The aim is to locate the discounted Steiner point $s=(x, y, z)$ to maximise the NPV. Let $l_{0}, l_{1}$ and $l_{2}$ be the construction lengths from $p_{0}, p_{1}$ and $p_{2}$ to $s$, respectively, which are given by Euclidean distances. The line segments $\mathrm{p}_{0} s, \mathrm{sp}_{1}$ and $s \mathrm{p}_{2}$ are called decline links.

A jumbo is a mobile carriage or platform fitted with mechanical arms upon which several drilling machines are mounted and is used to construct the decline and extract the ore. The ore at $p_{1}$ is extracted when the jumbo completes the construction of decline links $p_{0} s$ and $s p_{1}$. When the jumbo finishes the construction of all the remaining decline links, then the ore at $p_{2}$ is extracted.

First, we find the discounted cost for constructing a decline of length $l$. Let D, 
$\mathrm{C}$ and $\mathrm{d}$ be the decline development rate, development cost rate and discount rate, respectively. The time taken to construct $x$ distance of the decline is $x / D$. Therefore,

$$
\text { Discounted cost }=\int_{0}^{l} C r^{-x / D} d x=\frac{C D}{\log r}\left(1-r^{-l / D}\right),
$$

where $r=1+d$.

To construct the entire underground access network the decline links $p_{0} s$, $\mathrm{sp}_{1}$ and $\mathrm{sp}_{2}$ need to be developed. The discounted costs for constructing these decline links are expressed using equation (1) and with an appropriate time discount factor. The negative cash flows generated from the access development are

$$
\begin{aligned}
\mathrm{NPV}_{\mathrm{dev}} & =\frac{\mathrm{CD}}{\log r}\left[\left(\mathrm{r}^{-\mathrm{l}_{0} / \mathrm{D}}-1\right)+\mathrm{r}^{-\mathrm{l}_{0} / \mathrm{D}}\left(\mathrm{r}^{-\mathrm{l}_{1} / \mathrm{D}}-1\right)+\mathrm{r}^{-\left(\mathrm{l}_{0}+\mathrm{l}_{1}\right) / \mathrm{D}}\left(\mathrm{r}^{-\mathrm{l}_{2} / \mathrm{D}}-1\right)\right] \\
& =\frac{\mathrm{CD}}{\log \mathrm{r}}\left(\mathrm{r}^{-\left(\mathrm{l}_{0}+\mathrm{l}_{1}+\mathrm{l}_{2}\right) / \mathrm{D}}-1\right)
\end{aligned}
$$

The ore at point $p_{1}$ is extracted before $p_{2}$. Therefore, the total times taken to reach $p_{1}$ and $p_{2}$ are $t_{0}+t_{1}$ and $t_{0}+t_{1}+t_{2}$, respectively, where $t_{0}=l_{0} / D$, $t_{1}=l_{1} / D, t_{2}=l_{2} / D$. The positive NPV generated from the ore production is

$$
\mathrm{NPV}_{\text {pro }}=\mathrm{V}_{1} \mathrm{r}^{-\left(\mathrm{l}_{0}+\mathrm{l}_{1}\right) / \mathrm{D}}+\mathrm{V}_{2} \mathrm{r}^{-\left(\mathrm{l}_{0}+\mathrm{l}_{1}+\mathrm{l}_{2}\right) / \mathrm{D}} .
$$

We consider only the cash flows generated from ore production and access development because these directly relate to the location of the discounted Steiner point. Therefore, the total NPV is

$$
\mathrm{NPV}=\mathrm{NPV}_{\text {pro }}+\mathrm{NPV}_{\mathrm{dev}} .
$$

By substituting equations (2) and (3) into equation (4),

$$
N P V=V_{1} r^{-\left(l_{0}+l_{1}\right) / D}+\left(V_{2}+V_{c}\right) r^{-\left(l_{0}+l_{1}+l_{2}\right) / D}-V_{c},
$$


where $V_{c}=C D / \log r$. Equation (5) is the objective function for this problem. The problem we analyse is expressed as an optimisation problem:

$$
\begin{array}{ll}
\text { maximise: } & V_{1} r^{-\left(l_{0}+l_{1}\right) / D}+\left(V_{2}+V_{c}\right) r^{-\left(l_{0}+l_{1}+l_{2}\right) / D}-V_{c}, \\
\text { such that: } & l_{0}, l_{1}, l_{2} \geqslant 0 .
\end{array}
$$

The decision variables are $l_{0}, l_{1}$ and $l_{2}$ and they are functions of the discounted Steiner point coordinates $x, y$ and $z$. There are additional constraints on $l_{0}$, $l_{1}$ and $l_{2}$ that are imposed by the geometry of the network. Later, we obtain such a constraint, given by equation (14). A maximum always exists since the NPV is bounded above by $V_{1}+V_{2}$. The optimal location of the discounted Steiner point lies either in the interior or on the boundary of a triangle given by the three vertices $p_{0}, p_{1}$ and $p_{2}$. The objective function is differentiable on the problem domain with respect to $x, y$ and $z$, except at $p_{0}, p_{1}$ and $p_{2}$. The problem is a non-convex problem, therefore methods that assume convexity cannot be used.

The derivatives of the objective function with respect to $x, y$ and $z$ are set equal to zero

$$
\begin{aligned}
& (A+B)\left(\frac{\partial l_{0}}{\partial x}+\frac{\partial l_{1}}{\partial x}\right)+B \frac{\partial l_{2}}{\partial x}=0, \\
& (A+B)\left(\frac{\partial l_{0}}{\partial y}+\frac{\partial l_{1}}{\partial y}\right)+B \frac{\partial l_{2}}{\partial y}=0, \\
& (A+B)\left(\frac{\partial l_{0}}{\partial z}+\frac{\partial l_{1}}{\partial z}\right)+B \frac{\partial l_{2}}{\partial z}=0,
\end{aligned}
$$

where $A=V_{1} \log r / D$ and $B=\left(V_{2} \log r / D+C\right) r^{-l_{2} / D}$. Equations (6), and (8) are expressed in terms of gradients,

$$
(A+B) \nabla\left(l_{0}+l_{1}\right)+B \nabla l_{2}=0 .
$$

Next, we develop an important property of the angles at the discounted Steiner point. 

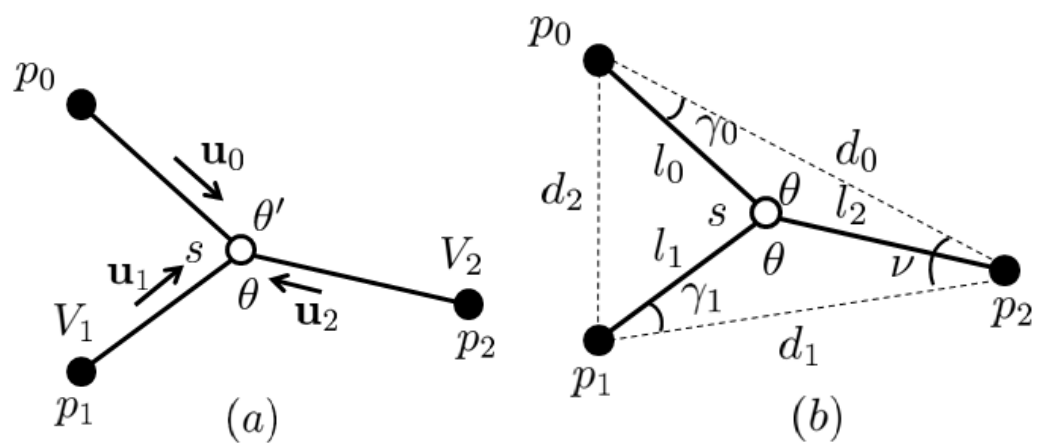

Figure 2: The (a) vector and (b) geometric representation of the problem.

Let $\mathbf{u}_{0}, \mathbf{u}_{1}$ and $\mathbf{u}_{2}$ be the unit vectors which are directed from the fixed points $p_{0}, p_{1}$ and $p_{2}$ towards $s$, respectively. Let $\theta$ be the angle between $\mathbf{u}_{1}$ and $\mathbf{u}_{2}$, and $\theta^{\prime}$ be the angle between $\mathbf{u}_{0}$ and $\mathbf{u}_{2}$, as shown in Figure 2(a). The unit vectors are expressed as the gradients, $\mathbf{u}_{0}=\nabla l_{0}, \mathbf{u}_{1}=\nabla l_{1}$, $\mathbf{u}_{2}=\nabla l_{2}$, and after substituting these into equation (9) we have

$$
(A+B)\left(\mathbf{u}_{0}+\mathbf{u}_{1}\right)+B \mathbf{u}_{2}=0 .
$$

After taking the dot product of equation (10) with $\left(\mathbf{u}_{0}-\mathbf{u}_{1}\right)$,

$$
(A+B)\left(\mathbf{u}_{0}+\mathbf{u}_{1}\right) \cdot\left(\mathbf{u}_{0}-\mathbf{u}_{1}\right)+B \mathbf{u}_{2} \cdot\left(\mathbf{u}_{0}-\mathbf{u}_{1}\right)=0 .
$$

The expression above reduces to $\mathbf{u}_{2} \cdot \mathbf{u}_{0}=\mathbf{u}_{2} \cdot \mathbf{u}_{1}$. Therefore, $\cos \theta=\cos \theta^{\prime}$ and $\theta=\theta^{\prime}$.

Now, we develop the discount equation which is a relationship between $l_{2}$ and $\theta$ in terms of $V_{1}, V_{2}$ and the discount rate. After taking the dot product of equation (10) with $\mathbf{u}_{2}$ we obtain

$$
(A+B)\left(\mathbf{u}_{0}+\mathbf{u}_{1}\right) \cdot \mathbf{u}_{2}+B \mathbf{u}_{2} \cdot \mathbf{u}_{2}=0 .
$$

Since $\mathbf{u}_{0} \cdot \mathbf{u}_{2}=\mathbf{u}_{1} \cdot \mathbf{u}_{2}=\cos \theta$ and $\mathbf{u}_{2} \cdot \mathbf{u}_{2}=1$ we obtain

$$
\cos \theta=-\frac{1}{2(A / B+1)}=-\frac{1}{2\left(\mathrm{kr}^{\mathrm{l}} / \mathrm{D}+1\right)} .
$$


where $\mathrm{k}=\mathrm{V}_{1} /\left[\mathrm{V}_{2}+\mathrm{CD} / \log \mathrm{r}\right]$ is a dimensionless constant.

We consider two special cases, a zero and an infinite discount rate which correspond to the upper and lower bounds for angle $\theta$, respectively. If $d=0$, then $r=1$ and $\theta=2 \pi / 3$, which corresponds to the classical Steiner location [2] and this angle is the upper bound of $\theta$. If $d \rightarrow \infty$, then $r \rightarrow \infty$ and $\theta=\pi / 2$. Hence, $\pi / 2$ is the lower bound of $\theta$.

Thus far, we have derived equation (12) in terms of $\theta$ and $l_{2}$ using the objective function. Now, we relate $\theta$ and $l_{2}$ geometrically and generate another equation which we define as the geometric equation.

Let $\gamma_{0}=\angle s p_{0} p_{2}, \gamma_{1}=\angle s p_{1} p_{2}$, and $d_{0}, d_{1}$ and $d_{2}$ be the Euclidean distances as shown in Figure 2(b). Let $v=\angle p_{0} p_{2} p_{1}$. Hence, by the cosine rule,

$$
v=\arccos \left(\frac{\mathrm{d}_{0}^{2}+\mathrm{d}_{1}^{2}-\mathrm{d}_{2}^{2}}{2 \mathrm{~d}_{0} \mathrm{~d}_{1}}\right) .
$$

By applying the sine rule to triangles $p_{0} p_{2} s$ and $s p_{1} p_{2}$ we obtain $\gamma_{0}$ and $\gamma_{1}$, respectively. By setting the sum of the angles in the quadrilateral $p_{0} p_{2} p_{1} s$ equal to $2 \pi$ and substituting the values for $\gamma_{1}$ and $\gamma_{0}$, the geometric equation is

$$
l_{2}=\frac{d_{0} d_{1}|\sin (2 \theta+v)|}{\sin \theta \sqrt{d_{0}^{2}+d_{1}^{2}+2 \cos (2 \theta+v) d_{0} d_{1}}} .
$$

\section{The discounted Steiner point algorithm}

In the previous section, the discount and geometric equations were derived in terms of $\theta$ and $l_{2}$. We now propose an iterative process to find the optimal values for $\theta$ and $l_{2}$. Once these two parameters are known the coordinates of the discounted Steiner point are calculated.

Let $\theta^{*}$ and $l_{2}^{*}$ be the optimal values obtained from the iterative process. Then optimal values $l_{0}^{*}$ and $l_{1}^{*}$ are calculated by applying the sine rule for triangles 
$p_{0} s p_{2}$ and $p_{1} s p_{2}$ in Figure 2(b). The maximum NPV is

$$
\mathrm{NPV}^{*}=\mathrm{V}_{1} \mathrm{r}^{-\left(\mathrm{l}_{0}^{*}+l_{1}^{*}\right) / \mathrm{D}}+\left(\mathrm{V}_{2}+\mathrm{V}_{\mathrm{c}}\right) \mathrm{r}^{-\left(l_{0}^{*}+l_{1}^{*}+l_{2}^{*}\right) / \mathrm{D}}-\mathrm{V}_{\mathrm{c}} .
$$

Since the distances $l_{0}^{*}, l_{1}^{*}$ and $l_{2}^{*}$ are known, $x, y$ and $z$ are calculated by solving three quadratic simultaneous equations:

$$
\begin{aligned}
& l_{0}^{* 2}=\left(x_{0}-x\right)^{2}+\left(y_{0}-y\right)^{2}+\left(z_{0}-z\right)^{2} \\
& l_{1}^{* 2}=\left(x-x_{1}\right)^{2}+\left(y-y_{1}\right)^{2}+\left(z-z_{1}\right)^{2} \\
& l_{2}^{* 2}=\left(x-x_{2}\right)^{2}+\left(y-y_{2}\right)^{2}+\left(z-z_{2}\right)^{2}
\end{aligned}
$$

Based on many numerical trials, the process always appears to converge rapidly and the results are independent of the initial value of $\theta$. These trials also show that the optimal solution converges uniquely. Algorithm 1 describes the numerical procedure where $\epsilon$ is the accepted error.

\section{$4 \quad$ Numerical trials}

We now give some results of numerical trials, obtain using

$$
\begin{aligned}
& \mathrm{V}_{1}=\$ 60 \mathrm{M}, \quad \mathrm{V}_{2}=\$ 40 \mathrm{M}, \quad \mathrm{C}=\$ 6000 / \mathrm{m}, \quad \mathrm{D}=1560 \mathrm{~m} / \text { year}, \\
& \mathrm{d}=0,5,10,20,50,100, \infty \% / \text { year, } \\
& \mathrm{p}_{0}=(200,1200,1000), \quad \mathrm{p}_{1}=(0,0,0), \quad \mathrm{p}_{2}=(1000,1000,0),
\end{aligned}
$$

with coordinates given in metres.

We showed analytically for zero and infinite discount rates that the corresponding optimal values of $\theta$ are $2 \pi / 3$ and $\pi / 2$, respectively. The results of the trials confirm this, as shown in Table 1 . Figure 3 shows that $p_{1}$ is accessed sooner for higher discount rates when the optimal location of the discounted Steiner point is used. However, the distance from the discounted Steiner point to $p_{2}$ increases. In addition, when the discount rate is increased 


\section{Algorithm 1: Discounted Steiner Point algorithm.}

Input: $V_{1}, V_{2}, C, D, d, \epsilon$ and locations of $p_{0}, p_{1}$ and $p_{2}$

Output: The location of the discounted Steiner point and NPV

1 Calculate Euclidean distances $d_{0}, d_{1}$ and $v$ using equation (13)

2 Initialisation: $\theta(0)=2 \pi / 3$

$3 i=0$

4 repeat

5 Compute $l_{2}$ :

$$
l_{2}(i+1)=\frac{d_{0} d_{1}|\sin (2 \theta(i)+v)|}{\sin \theta(i) \sqrt{d_{0}^{2}+d_{1}^{2}+2 \cos (2 \theta(i)+v) d_{0} d_{1}}}
$$

Update $\theta$ :

$$
\theta(i+1)=\arccos \left(-\frac{1}{2\left(\mathrm{kr}^{\mathrm{l}}(\mathrm{i}+1) / \mathrm{D}+1\right)}\right)
$$

$6 \quad i=i+1$

7 until $|\theta(i)-\theta(i-1)|<\epsilon$

$8 \theta^{*}=\theta(\mathfrak{i})$ and $l_{2}^{*}=l_{2}(\mathfrak{i})$

9 The optimal NPV is given by equation (15)

10 The optimal location of the discounted Steiner point is calculated using equations (16), (17) and (18)

the NPV is reduced. For an infinite discount rate, the optimal location of the discounted Steiner point is on the edge $p_{0} p_{1}$, which also gives the minimum sum of the construction length for the $p_{0} s$ and $s p_{1}$ decline links.

The last column of Table 1 shows the improvement in NPV when the discounted Steiner point algorithm is applied compared with placing the discounted Steiner point at the classical position $(\theta=2 \pi / 3)$. The discounted Steiner point algorithm gives an improvement for all discount rates and greater improvements as the discount increases. 


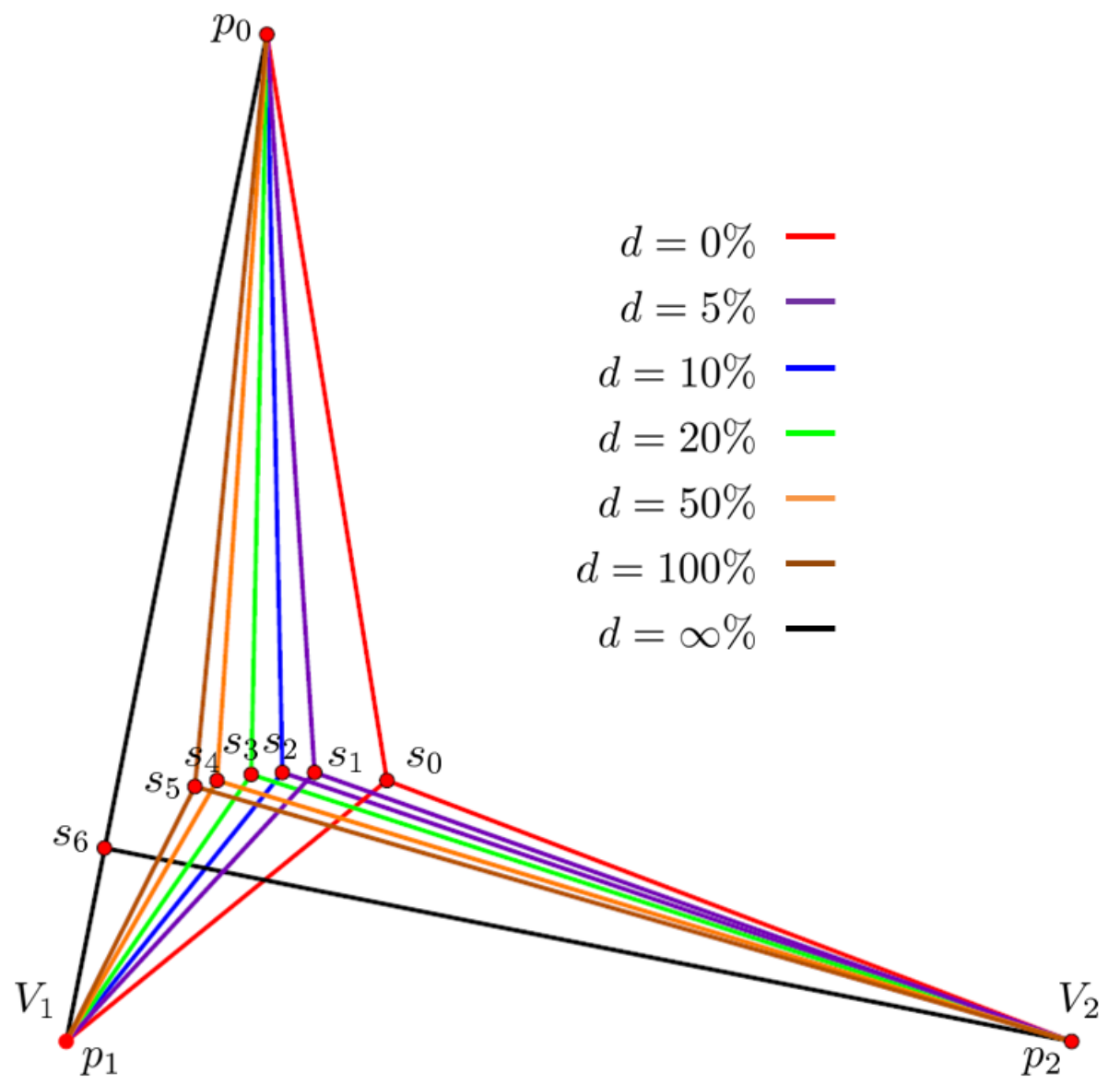

Figure 3: The optimal locations of the discounted Steiner point. 
Table 1: Variation of the NPV for a range of discount rates (DR) per year.

\begin{tabular}{rrrrrr} 
DR & Steiner point & $\theta^{\circ}$ & $l_{2}(\mathrm{~m})$ & $\mathrm{NPV}(\$ \mathrm{~K})$ & Improvement $(\$ \mathrm{~K})$ \\
\hline 0 & $\mathrm{~s}_{0}=(319,578,259)$ & 120 & 728 & 88660 & 0 \\
5 & $\mathrm{~s}_{1}=(247,514,267)$ & 113 & 798 & 84618 & 49 \\
10 & $\mathrm{~s}_{2}=(215,482,267)$ & 110 & 828 & 80997 & 159 \\
20 & $\mathrm{~s}_{3}=(184,449,265)$ & 106 & 857 & 74700 & 393 \\
50 & $\mathrm{~s}_{4}=(150,409,259)$ & 103 & 888 & 60875 & 958 \\
100 & $\mathrm{~s}_{5}=(128,381,253)$ & 100 & 907 & 46971 & 1516 \\
$\infty$ & $\mathrm{s}_{6}=(38,230,192)$ & 90 & 980 & 0 & 0
\end{tabular}

\section{Conclusion and future research}

We developed an algorithm to optimally locate a discounted Steiner point. The discounted Steiner point algorithm provides higher NPV compared with the placement of the discounted Steiner point at the classical position. Furthermore, we showed that paths from the discounted Steiner point to the surface portal and the first resource point make equal angles with the path from the discounted Steiner point to the second resource point.

Future work will be to locate a discounted Steiner point with more realistic constraints, such as the gradient constraint. The gradient constraint on the decline is the most important physical constraint on the access network and defines a safe climbing limit for trucks. We then plan to extend the discounted Steiner point algorithm to optimally locate multiple discounted Steiner points.

Acknowledgements: This work was supported by Rand Mining and Tribune Resources and we thank Dr John Andrews for his valuable comments and sharing his knowledge. This work is funded by a Gilbert Rigg scholarship and an ARC Linkage grant. 


\section{References}

[1] K. F. Lane, The economic definition of ore-cutoff grade in theory and practice. Mining Journal Books Limited, London 1988. C317

[2] F. K. Hwang, D. S. Richards and P. Winter, The Steiner tree problem. Elsevier, 1992. http://www.elsevier.com/books/ the-steiner-tree-problem/hwang/978-0-444-89098-6 C317, C322

[3] M. Brazil and D. A. Thomas, Network optimisation for the design of underground mines. Networks 49:40-50, 2007. doi:10.1002/net.20140 C317

[4] M. Brazil, J. H. Rubinstein, D. A. Thomas, J. F. Weng and N. C. Wormald, Gradient-constrained minimum networks (I). Fundamentals. J. Global Optim. 21:139-155, 2001. doi:10.1023/A:1011903210297 C317

\section{Author addresses}

1. K. G. Sirinanda, Department of Mechanical Engineering, The University of Melbourne, Australia. mailto:kash.s@student.unimelb.edu.au

2. M. Brazil, Department of Electrical and Electronic Engineering, The University of Melbourne, Australia. mailto:brazil@unimelb.edu . au

3. P. A. Grossman, Department of Mechanical Engineering, The University of Melbourne, Australia. mailto:peterag@unimelb.edu.au

4. J. H. Rubinstein, Department of Mathematics and Statistics, The University of Melbourne, Australia. mailto:rubin@ms . unimelb.edu . au 
5. D. A. Thomas, Department of Mechanical Engineering, The University of Melbourne, Australia. mailto:doreen.thomas@unimelb.edu . au 